Some common fixed point theorems for a class of fuzzy contractive mappings

\author{
M. A. Ahmed
}




\title{
SOME COMMON FIXED POINT THEOREMS FOR A CLASS OF FUZZY CONTRACTIVE MAPPINGS
}

\author{
M. A. AHMED
}

Received 11 November, 2004

\begin{abstract}
The purpose of this paper is to state and prove a new lemma generalizing Lemma 3.1 of Arora and Sharma [1] and Proposition 3.2 of Lee and Cho [10]. Some common fixed point theorems for a type of fuzzy contractive mappings are also established. These theorems extend and generalize several previous results $[3,14,21,22]$.
\end{abstract}

1991 Mathematics Subject Classification: 47H10, 54H25

Keywords: fuzzy sets, fuzzy map, fuzzy contractive mappings, common fixed points.

\section{INTRODUCTION}

Common fixed point theorems have been applied to diverse problems during the last few decades. These theorems provide techniques for solving a variety of applied problems in mathematical science and in dynamic programming (see, e. g., $[4,15,16])$. Extensions of the Banach contraction principle to multivalued mappings were initiated independently by Markin [11] and Nadler [13]. Therefore, results on fixed points of contractive type multivalued mappings have been carried out by many authors (see, for example, [2,17,21]).

The theory of fuzzy sets was investigated by Zadeh [24] in 1965. Some applications on results in this theory are discussed (see [9,23]). In 1981, Heilpern [7] first introduced the concept of fuzzy contractive mappings and proved a fixed point theorem for these mappings in metric linear spaces. His result is a generalization of the fixed point theorem for point-to-set maps of Nadler [13]. Later, several fixed point theorems for types of fuzzy contractive mappings appeared (see, for instance, $[1,18-20])$

In this paper, we state and prove a new lemma generalizing Lemma 3.1 of Arora and Sharma [1] and Proposition 3.2 of Lee and Cho [10]. Two common fixed point theorems of a type of fuzzy contractive mappings are established. These theorems generalize and extend results in $[3,14,21,22]$. Finally, we state a conclusion containing a brief of our results and future research. 


\section{BASIC PRELIMINARIES}

The definitions and terminologies for further discussions are taken from Heilpern [7]. Let $(X, d)$ be a metric linear space. A fuzzy set in $X$ is a function with domain $X$ and values in [0,1]. If $A$ is a fuzzy set and $x \in X$, then the function-value $A(x)$ is called the grade of membership of $x$ in $A$. The collection of all fuzzy sets in $X$ is denoted by $\mathcal{F}(X)$.

Let $A \in \mathcal{F}(X)$ and $\alpha \in[0,1]$. The $\alpha$-level set of $A$, denoted by $A_{\alpha}$, is defined by the formula

$$
A_{\alpha}= \begin{cases}\{x: A(x) \geq \alpha\} & \text { if } \alpha \in(0,1], \\ \{x: A(x)>0\} & \text { if } \alpha=0 .\end{cases}
$$

where $\bar{B}$ is the closure of a (nonfuzzy) set $B$.

Definition 1. A fuzzy set $A$ in $X$ is an approximate quantity if and only if its $\alpha$ level set is a nonempty compact convex (nonfuzzy) subset of $X$ for each $\alpha \in[0,1]$ and $\sup _{x \in X} A(x)=1$.

The set of all approximate quantities, denoted $W(X)$, is a subcollection of $\mathcal{F}(X)$.

Definition 2. Let $A, B \in W(X), \alpha \in[0,1]$ and $C P(X)$ be the set of all nonempty compact subsets of $X$. Then one puts $p_{\alpha}(A, B)=\inf _{x \in A_{\alpha}, y \in B_{\alpha}} d(x, y), \delta_{\alpha}(A, B)=$ $\sup _{x \in A_{\alpha}, y \in B_{\alpha}} d(x, y)$, and $D_{\alpha}(A, B)=H\left(A_{\alpha}, B_{\alpha}\right)$, where $H$ is the Hausdorff metric between two sets in the collection $C P(X)$.

We define the functions $p(A, B)=\sup _{\alpha} p_{\alpha}(A, B), \delta(A, B)=\sup _{\alpha} \delta_{\alpha}(A, B)$, and $D(A, B)=\sup _{\alpha} D_{\alpha}(A, B)$.

Note that $p_{\alpha}$ is nondecreasing function of $\alpha$.

Definition 3. Let $A, B \in W(X)$. Then $A$ is said to be more accurate than $B$ (or $B$ includes $A$ ), denoted by $A \subset B$, if and only if $A(x) \leq B(x)$ for each $x \in X$.

The relation $\subset$ induces a partial ordering on $W(X)$.

Definition 4. Let $X$ be an arbitrary set and $Y$ be a metric linear space. $F$ is said to be a fuzzy mapping if and only if $F$ is a mapping from the set $X$ into $W(Y)$, i. e., $F(x) \in W(Y)$ for each $x \in X$.

The following lemma and proposition are used in the sequel.

Lemma 1 ([12]). Suppose that $\gamma:[0, \infty) \rightarrow[0, \infty)$ is a right continuous function such that $\gamma(t)<t$ for all $t>0$. Then for every $t>0, \lim _{n \rightarrow \infty} \gamma^{n}(t)=0$, where $\gamma^{n}$ is the nth iterate of $\gamma, n \in \mathbb{N} \cup\{0\}$ * $^{*}$

Proposition 1 ([13]). If $A, B \in C P(X)$ and $a \in A$, then there exists $b \in B$ such that $d(a, b) \leq H(A, B)$.

\footnotetext{
${ }^{*} \mathbb{N}$ is the set of all positive integers
} 
We consider the set $\Phi$ of all functions $\phi:[0, \infty)^{5} \rightarrow[0, \infty)$ with the following properties:

(i) $\phi$ is nondecreasing with respect to each variable,

(ii) $\phi$ is right continuous with respect to each variable,

(iii) for each $t>0, \Psi(t)=\max \{\phi(t, t, t, t, t), \phi(t, t, t, 2 t, 0), \phi(t, t, t, 0,2 t)\}<t$.

\section{MAIN RESUltS}

Throughout this paper, let $(X, d)$ be a metric space. We consider a subcollection of $\mathcal{F}(X)$ denoted by $W^{*}(X)$. Each fuzzy set $A \in W^{*}(x)$, its $\alpha$-level set is a nonempty compact (nonfuzzy) subset of $X$ for each $\alpha \in[0,1]$. It is obvious that each element $A \in W(X)$ leads one to $A \in W^{*}(X)$ but the converse is not true. Now, we introduce the improvements of the lemmas in Heilpern [7] as follows.

Lemma 2. If $\left\{x_{0}\right\} \subset A$ for each $A \in W^{*}(X)$ and $x_{0} \in X$, then $p_{\alpha}\left(x_{0}, B\right) \leq$ $D_{\alpha}(A, B)$ for each $B \in W^{*}(X)$.

Lemma 3. $p_{\alpha}(x, A) \leq d(x, y)+p_{\alpha}(y, A)$ for all $x, y \in X$ and $A \in W^{*}(X)$.

Lemma 4. Let $x \in X, A \in W^{*}(X)$ and $\{x\}$ be a fuzzy set with membership function equal to a characteristic function of the set $\{x\}$. Then $\{x\} \subset A$ if and only if $p_{\alpha}(x, A)=0$ for each $\alpha \in[0,1]$.

Proof. If $\{x\} \subset A$, then $x \in A_{\alpha}$ for each $\alpha \in[0,1]$. This implies that $p_{\alpha}(x, A)=$ $\inf _{y \in A_{\alpha}} d(x, y)=0$ for any $\alpha \in[0,1]$. Conversely, if $p_{\alpha}(x, A)=0$, then we have $\inf _{y \in A_{\alpha}} d(x, y)=0$. It follows that $x \in \bar{A}_{\alpha}=A_{\alpha}$ for an arbitrary $\alpha \in[0,1]$. Then $\{x\} \subset A$.

Also, we state and prove a new lemma in the following way.

Lemma 5. Let $(X, d)$ be a complete metric space, $F: X \rightarrow W^{*}(X)$ be a fuzzy map and $x_{0} \in X$. Then there exists $x_{1} \in X$ such that $\left\{x_{1}\right\} \subset F\left(x_{0}\right)$.

Proof. For $n \in \mathbb{N},\left(\left(F\left(x_{0}\right)\right)_{n /(n+1)}\right)$ is a decreasing sequence of nonempty compact subsets of $X$. Thus we have from Proposition 11.4 and Remark 11.5 of [25, pp. 495-496] that $\bigcap_{n=1}^{\infty}\left(F\left(x_{0}\right)\right)_{n /(n+1)}$ is nonempty and compact.

Let $x_{1} \in \bigcap_{n=1}^{\infty}\left(F\left(x_{0}\right)\right)_{n /(n+1)}$. Then $\frac{n}{n+1} \leq\left(F\left(x_{0}\right)\right)\left(x_{1}\right) \leq 1$. As $n \rightarrow \infty$, we get that $\left(F\left(x_{0}\right)\right)\left(x_{1}\right)=1$. This implies that $\left\{x_{1}\right\} \subset F\left(x_{0}\right)$.

Remark 1. It is clear that Lemma 5 is a generalization of Lemma 3.1 of Arora and Sharma [1] and Proposition 3.2 of Lee and Cho [10].

Now, we are ready to prove our main theorems. 
Theorem 1. Let $(X, d)$ be a complete metric space and $F_{1}, F_{2}$ be fuzzy mappings from $X$ into $W^{*}(X)$. If there is a $\phi \in \Phi$ such that for all $x, y \in X$,

$$
\begin{aligned}
& D\left(F_{1}(x), F_{2}(y)\right) \leq \phi\left(d(x, y), p\left(x, F_{1}(x)\right),\right. \\
& \left.\quad p\left(y, F_{2}(y)\right), p\left(x, F_{2}(y)\right), p\left(y, F_{1}(x)\right)\right),
\end{aligned}
$$

then there exists $z \in X$ such that $\{z\} \subset F_{1}(z)$ and $\{z\} \subset F_{2}(z)$.

Proof. Let $x_{0} \in X$. Then by Lemma 5, there exists $x_{1} \in X$ such that $\left\{x_{1}\right\} \subset$ $F_{1}\left(x_{0}\right)$. For $x_{1} \in X$, the set $\left(F_{2}\left(x_{1}\right)\right)_{1}$ is nonempty compact subset of $X$. Since $\left(F_{1}\left(x_{0}\right)\right)_{1}$ and $\left(F_{2}\left(x_{1}\right)\right)_{1}$ belong to $C P(X)$ and $x_{1} \in\left(F_{1}\left(x_{0}\right)\right)_{1}$, Proposition 1 asserts that there exists $x_{2} \in\left(F_{2}\left(x_{1}\right)\right)_{1}$ such that $d\left(x_{1}, x_{2}\right) \leq D_{1}\left(F_{1}\left(x_{0}\right), F_{2}\left(x_{1}\right)\right)$. So, we have from Lemma 4 and the property (i) of $\phi$ that

$$
\begin{aligned}
d\left(x_{1}, x_{2}\right) \leq & D_{1}\left(F_{1}\left(x_{0}\right), F_{2}\left(x_{1}\right)\right) \leq D\left(F_{1}\left(x_{0}\right), F_{2}\left(x_{1}\right)\right) \\
\leq & \phi\left(d\left(x_{0}, x_{1}\right),\right. \\
& \left.\quad p\left(x_{0}, F_{1}\left(x_{0}\right)\right), p\left(x_{1}, F_{2}\left(x_{1}\right)\right), p\left(x_{0}, F_{2}\left(x_{1}\right)\right), p\left(x_{1}, F_{1}\left(x_{0}\right)\right)\right) \\
\leq & \phi\left(d\left(x_{0}, x_{1}\right), d\left(x_{0}, x_{1}\right), d\left(x_{1}, x_{2}\right), d\left(x_{0}, x_{1}\right)+d\left(x_{1}, x_{2}\right), 0\right) .
\end{aligned}
$$

If $d\left(x_{1}, x_{2}\right)>d\left(x_{0}, x_{1}\right)$, then

$$
d\left(x_{1}, x_{2}\right) \leq \phi\left(d\left(x_{1}, x_{2}\right), d\left(x_{1}, x_{2}\right), d\left(x_{1}, x_{2}\right), 2 d\left(x_{1}, x_{2}\right), 0\right)<d\left(x_{1}, x_{2}\right) .
$$

This contradiction demands that

$$
d\left(x_{1}, x_{2}\right) \leq \phi\left(d\left(x_{0}, x_{1}\right), d\left(x_{0}, x_{1}\right), d\left(x_{0}, x_{1}\right), 2 d\left(x_{0}, x_{1}\right), 0\right) .
$$

Similarly, one can deduce that

$$
d\left(x_{2}, x_{3}\right) \leq \phi\left(d\left(x_{1}, x_{2}\right), d\left(x_{1}, x_{2}\right), d\left(x_{1}, x_{2}\right), 0,2 d\left(x_{1}, x_{2}\right)\right) .
$$

By induction, we have a sequence $\left(x_{n}\right)$ of points in $X$ such that, for all $n \in \mathbb{N} \cup\{0\}$,

$$
\left\{x_{2 n+1}\right\} \subset F_{1}\left(x_{2 n}\right), \quad\left\{x_{2 n+2}\right\} \subset F_{2}\left(x_{2 n+1}\right) .
$$

It follows by induction that $d\left(x_{n}, x_{n+1}\right) \leq \Psi^{n}\left(d\left(x_{0}, x_{1}\right)\right)$, where $\Psi$ is defined in the property (iii) of $\phi$. Then, Lemma 1 gives that $\lim _{n \rightarrow \infty} d\left(x_{n}, x_{n+1}\right)=0$. Since

$$
d\left(x_{n}, x_{m}\right) \leq d\left(x_{n}, x_{n+1}\right)+d\left(x_{n+1}, x_{n+2}\right)+\ldots+d\left(x_{m-1}, x_{m}\right),
$$

then $\lim _{n, m \rightarrow \infty} d\left(x_{n}, x_{m}\right)=0$. Therefore, $\left(x_{n}\right)$ is a Cauchy sequence. Since $X$ is a complete metric space, then there exists $z \in X$ such that $\lim _{n \rightarrow \infty} x_{n}=z$. Next, we show that $\{z\} \subset F_{i}(z), i=1,2$. Now, we get from Lemma 2 and Lemma 3 that

$$
\begin{aligned}
p_{\alpha}\left(z, F_{2}(z)\right) \leq d\left(z, x_{2 n+1}\right)+p_{\alpha}\left(x_{2 n+1},\right. & \left.F_{2}(z)\right) \\
& \leq d\left(z, x_{2 n+1}\right)+D_{\alpha}\left(F_{1}\left(x_{2 n}\right), F_{2}(z)\right),
\end{aligned}
$$


for each $\alpha \in[0,1]$. Taking the supremum on $\alpha$ in the last inequality, we obtain from the property (i) of $\phi$ that

$$
\begin{aligned}
p\left(z, F_{2}(z)\right) \leq & d\left(z, x_{2 n+1}\right)+D\left(F_{1}\left(x_{2 n}\right), F_{2}(z)\right) \\
\leq & d\left(z, x_{2 n+1}\right)+\phi\left(d\left(x_{2 n}, z\right), p\left(x_{2 n}, F_{1}\left(x_{2 n}\right)\right), p\left(z, F_{2}(z)\right),\right. \\
& \left.p\left(x_{2 n}, F_{2}(z)\right), p\left(z, F_{1}\left(x_{2 n}\right)\right)\right) \\
\leq & d\left(z, x_{2 n+1}\right)+\phi\left(d\left(x_{2 n}, z\right), d\left(x_{2 n}, x_{2 n+1}\right), p\left(z, F_{2}(z)\right),\right. \\
& \left.p\left(x_{2 n}, F_{2}(z)\right), d\left(z, x_{2 n+1}\right)\right) .
\end{aligned}
$$

As $n \rightarrow \infty$, we have from the properties (i), (ii) and (iii) of $\phi$ with $p\left(z, F_{2}(z)\right) \neq 0$ that

$$
\begin{aligned}
p\left(z, F_{2}(z)\right) & \leq \phi\left(0,0, p\left(z, F_{2}(z)\right), p\left(z, F_{2}(z)\right), 0\right) \\
& \leq \phi\left(p\left(z, F_{2}(z)\right), p\left(z, F_{2}(z)\right), p\left(z, F_{2}(z)\right), p\left(z, F_{2}(z)\right), p\left(z, F_{2}(z)\right)\right) \\
& <p\left(z, F_{2}(z)\right) .
\end{aligned}
$$

This contradiction yields $p\left(z, F_{2}(z)\right)=0$. We then get from Lemma 4 that $\{z\} \subset$ $F_{2}(z)$. Similarly, one can show that $\{z\} \subset F_{1}(z)$.

Example 1. Let $X=[0,1]$ endowed with the metric $d$ defined by $d(x, y)=|x-y|$. It is clear that $(X, d)$ is a complete metric space. Assume that $\phi\left(t_{1}, t_{2}, t_{3}, t_{4}, t_{5}\right)=\frac{3}{4} t_{1}$ for arbitrary $t_{i} \in[0, \infty), i=\overline{1,5}$. It is obvious that $\Psi(t)<t$ for all $t>0$. Let $F_{1}=F_{2}=F$. Define a fuzzy mapping $F$ on $X$ such that for all $x \in X, F(x)$ is the characteristic function for $\left\{\frac{3}{4} x\right\}$. For each $x, y \in X$,

$$
\begin{aligned}
& D(F(x), F(y))=\frac{3}{4} d(x, y) \\
& \quad=\phi(d(x, y), p(x, F(x)), p(y, F(y)), p(x, F(y)), p(y, F(x))) .
\end{aligned}
$$

The characteristic function for $\{0\}$ is the fixed point of $F$.

As corollaries of Theorem 1, we get the following statements.

Corollary 1. Let $(X, d)$ be a complete metric space and $F_{1}, F_{2}$ be fuzzy mappings from $X$ into $W^{*}(X)$ satisfying the following conditions: for any $x, y$ in $X$,

$$
\begin{aligned}
D\left(F_{1}(x), F_{2}(y)\right) \leq a_{1} p\left(x, F_{1}(x)\right)+a_{2} p(y & \left., F_{2}(y)\right)+a_{3} p\left(y, F_{1}(x)\right) \\
& +a_{4} p\left(y, F_{1}(x)\right)+a_{5} d(x, y),
\end{aligned}
$$

where $a_{1}, a_{2}, a_{3}, a_{4}$, and $a_{5}$ are non-negative real numbers, $\sum_{j=1}^{5} a_{i}<1$ and $a_{1}=$ $a_{2}$ or $a_{3}=a_{5}$. Then there exists $z \in X$ such that $\{z\} \subset F_{1}(z)$ and $\{z\} \subset F_{2}(z)$.

Proof. We consider the function $\phi:[0, \infty)^{5} \rightarrow[0, \infty)$ defined by the formula

$$
\phi\left(x_{1}, x_{2}, x_{3}, x_{4}, x_{5}\right)=a_{1} x_{2}+a_{2} x_{3}+a_{3} x_{5}+a_{4} x_{4}+a_{5} x_{1},
$$

where $\sum_{i=1}^{i=5} a_{i}<1$ such that $a_{1}=a_{2}$ or $a_{3}=a_{4}$. Since $\phi \in \Phi$, we have from Theorem 1 that there exists $z \in X$ such that $\{z\} \subset F_{1}(z)$ and $\{z\} \subset F_{2}(z)$. 
The following corollary is a fuzzy version of the fixed point theorem of Singh and Whitfield [21] for multivalued mappings.

Corollary 2. Let $(X, d)$ be a complete metric space and $F_{1}, F_{2}$ be fuzzy mappings from $X$ into $W^{*}(X)$. If there is a constant $\alpha, 0 \leq \alpha<1$, such that, for each $x, y \in X$,

$$
\begin{aligned}
D\left(F_{1}(x), F_{2}(y)\right) \leq \alpha \max \left\{d(x, y), \frac{1}{2}\left[p\left(x, F_{1}(x)\right)+p\left(y, F_{2}(y)\right)\right],\right. \\
\left.\frac{1}{2}\left[p\left(x, F_{2}(y)\right)+p\left(y, F_{1}(x)\right)\right]\right\},
\end{aligned}
$$

then there exists $z \in X$ such that $\{z\} \subset F_{1}(z)$ and $\{z\} \subset F_{2}(z)$.

Proof. We consider the function $\phi:[0, \infty)^{5} \rightarrow[0, \infty)$ defined by

$$
\phi\left(x_{1}, x_{2}, x_{3}, x_{4}, x_{5}\right)=\alpha \max \left\{x_{1}, \frac{1}{2}\left[x_{2}+x_{3}\right], \frac{1}{2}\left[x_{4}+x_{5}\right]\right\} .
$$

Since $\phi \in \Phi$, we get from Theorem 1 that there exists $z \in X$ such that $\{z\} \subset F_{1}(z)$ and $\{z\} \subset F_{2}(z)$.

Remark 2. (1) If there is a $\phi \in \Phi$ such that, for each $x, y \in X$,

$$
\begin{array}{r}
\delta\left(F_{1}(x), F_{2}(y)\right) \leq \phi\left(d(x, y), p\left(x, F_{1}(x)\right), p\left(y, F_{2}(y)\right),\right. \\
\left.p\left(x, F_{2}(y)\right), p\left(y, F_{1}(x)\right)\right),
\end{array}
$$

then the conclusion of Theorem 1 remains valid. This result is considered as a special case of Theorem 1 because $D\left(F_{1}(x), F_{2}(y)\right) \leq \delta\left(F_{1}(x), F_{2}(y)\right)$ [8, p. 414]. Moreover, this result generalizes Theorem 3.3 of Park and Jeong [14].

(2) Corollary 1 is [22, Theorem 3.1] without condition (a), where condition (a) reads as follows: "for each $x \in X$, there exists $\alpha(x) \in(0,1]$ such that $\left(F_{1}(x)\right)_{\alpha(x)}$ and $\left(F_{2}(x)\right)_{\alpha(x)}$ are nonempty closed bounded subsets of $\mathcal{F}(X)$." Also, Corollary 1 generalizes [3, Theorem 3.1].

(3) Theorems 3.1 and 3.4 of Park and Jeong [14] are special cases of Theorem 1.

The following theorem generalizes Theorem 1 to a sequence of fuzzy contractive mappings.

Theorem 2. Let $\left(F_{n}: n \in \mathbb{N} \cup\{0\}\right)$ be a sequence of fuzzy mappings from a complete metric space $(X, d)$ into $W^{*}(X)$. If there is a $\phi \in \Phi$ such that, for all $x, y \in X$,

$$
\begin{aligned}
& D\left(F_{0}(x), F_{n}(y)\right) \leq \phi\left(d(x, y), p\left(x, F_{0}(x)\right), p\left(y, F_{n}(y)\right),\right. \\
&\left.p\left(x, F_{n}(y)\right), p\left(y, F_{0}(x)\right)\right) \quad \forall(n \in \mathbb{N}),
\end{aligned}
$$

then there exists a common fixed point of the family $\left(F_{n}: n \in \mathbb{N} \cup\{0\}\right)$.

Proof. Putting $F_{1}=F_{0}$ and $F_{2}=F_{n}$ for all $n \in \mathbb{N}$ in Theorem 1. Then there exists a common fixed point of the family $\left(F_{n}: n \in \mathbb{N} \cup\{0\}\right)$. 
Remark 3. If there is a $\phi \in \Phi$ such that, for all $x, y \in X$,

$$
\begin{aligned}
& \delta\left(F_{0}(x), F_{n}(y)\right) \leq \phi\left(d(x, y), p\left(x, F_{0}(x)\right), p\left(y, F_{n}(y)\right),\right. \\
& \left.p\left(x, F_{n}(y)\right), p\left(y, F_{0}(x)\right)\right) \quad(\forall n \in \mathbb{N}),
\end{aligned}
$$

then the conclusion of Theorem 2 remains valid. This result is considered as a special case of Theorem 2 for the same reason as in Remark 2 (1).

\section{CONCLusion}

This paper presents an improvement of some results in $[1,7,10]$. Also, it presents two common fixed point theorems for a type of fuzzy contractive mappings. These theorems generalize and extend results in [3,14,22] and [21], respectively. A fixed point theorem for fuzzy contractive mappings is stated generalizing [1, Theorem 3.5]. Many applications of our main theorems are possible, e. g., for differential and integral equations. In view of the references [5,6], some future research can be done, for example:

(1) I believe that our results can be hold for $F C(X)$, where $F C(X)=\{A \in \mathcal{F}(X)$ : $A_{\alpha}$ is a nonempty closed (nonfuzzy) subset of $X$ for each $\left.\alpha \in[0,1]\right\}$,

(2) it is also possible to generalize our results to quasi-metric spaces.

\section{Acknowledgement}

I wish to thank Prof. B. E. Rhoades from the Indiana University (USA) and Dr. F. M. Zeyada for their critical reading of the manuscript and valuable comments.

\section{REFERENCES}

[1] S. C. Arora and V. Sharma, "Fixed point theorems for fuzzy mappings," Fuzzy Sets and Systems, vol. 110, no. 1, pp. 127-130, 2000.

[2] I. Beg and A. Azam, "Fixed points of asymptotically regular multivalued mappings," J. Austral. Math. Soc. Ser. A, vol. 53, no. 3, pp. 313-326, 1992.

[3] R. K. Bose and D. Sahani, "Fuzzy mappings and fixed point theorems," Fuzzy Sets and Systems, vol. 21, no. 1, pp. 53-58, 1987.

[4] P. Z. Daffer and H. Kaneko, "Application of $f$-contraction mappings to nonlinear integral equations," Bull. Inst. Math. Acad. Sinica, vol. 22, no. 1, pp. 69-74, 1994.

[5] M. Frigon and D. O'Regan, "Fuzzy contractive maps and fuzzy fixed points," Fuzzy Sets and Systems, vol. 129, no. 1, pp. 39-45, 2002.

[6] V. Gregori and S. Romaguera, "Fixed point theorems for fuzzy mappings in quasi-metric spaces," Fuzzy Sets and Systems, vol. 115, no. 3, pp. 477-483, 2000.

[7] S. Heilpern, "Fuzzy mappings and fixed point theorem," J. Math. Anal. Appl., vol. 83, no. 2, pp. $566-569,1981$.

[8] T. L. Hicks, "Multivalued mappings on probabilistic metric spaces," Math. Japon., vol. 46, no. 3, pp. 413-418, 1997.

[9] O. Kaleva, "Fuzzy differential equations," Fuzzy Sets and Systems, vol. 24, no. 3, pp. 301-317, 1987.

[10] B. S. Lee and S. J. Cho, "A fixed point theorem for contractive-type fuzzy mappings," Fuzzy Sets and Systems, vol. 61, no. 3, pp. 309-312, 1994. 
[11] J. T. Markin, “A fixed point theorem for set valued mappings,” Bull. Amer. Math. Soc., vol. 74, pp. 639-640, 1968.

[12] J. Matkowski, "Fixed point theorems for mappings with a contractive iterate at a point," Proc. Amer. Math. Soc., vol. 62, no. 2, pp. 344-348, 1977.

[13] S. B. Nadler, Jr., "Multi-valued contraction mappings," Pacific J. Math., vol. 30, pp. 475-488, 1969.

[14] J. Y. Park and J. U. Jeong, "Fixed point theorems for fuzzy mappings," Fuzzy Sets and Systems, vol. 87, no. 1, pp. 111-116, 1997.

[15] H. K. Pathak, "Application of fixed point theorems to abstract Volterra integrodifferential equations," Riv. Mat. Univ. Parma (5), vol. 3, no. 2, pp. 193-202 (1995), 1994.

[16] H. K. Pathak and B. Fisher, "Common fixed point theorems with applications in dynamic programming," Glas. Mat. Ser. III, vol. 31(51), no. 2, pp. 321-328, 1996.

[17] V. Popa, "Common fixed points for multifunctions satisfying a rational inequality," Kobe J. Math., vol. 2, no. 1, pp. 23-28, 1985.

[18] R. A. Rashwan and M. A. Ahmed, "Common fixed points of Greguš type multi-valued mappings," Arch. Math. (Brno), vol. 38, no. 1, pp. 37-47, 2002.

[19] B. E. Rhoades, "Fixed points of some fuzzy mappings," Soochow J. Math., vol. 22, no. 1, pp. 111-115, 1996.

[20] B. Singh and M. S. Chauhan, "Fixed points of associated multimaps of fuzzy maps," Fuzzy Sets and Systems, vol. 110, no. 1, pp. 131-134, 2000.

[21] K. L. Singh and J. H. M. Whitfield, "Fixed points for contractive type multivalued mappings," Math. Japon., vol. 27, no. 1, pp. 117-124, 1982.

[22] P. Vijayaraju and M. Marudai, "Fixed point theorems for fuzzy mappings," Fuzzy Sets and Systems, vol. 135, no. 3, pp. 401-408, 2003.

[23] C. Wu and G. Wang, "Convergence of sequences of fuzzy numbers and fixed point theorems for increasing fuzzy mappings and application," Fuzzy Sets and Systems, vol. 130, no. 3, pp. 383-390, 2002, theme: Fuzzy intervals.

[24] L. A. Zadeh, "Fuzzy sets," Information and Control, vol. 8, pp. 338-353, 1965.

[25] E. Zeidler, Nonlinear functional analysis and its applications. I. New York: Springer-Verlag, 1986, fixed-point theorems, Translated from the German by Peter R. Wadsack.

\section{Author's address}

\section{A. Ahmed}

Assiut University Department of Mathematics, Assiut 71516, Egypt

Current address: Teachers College, Department of Mathematics, P. O. Box 4341, Riyadh 11491, Kingdom of Saudi Arabia

E-mail address: mahmed68@yahoo.com 